\section{Protocolo de profilaxis antibiótica para pacientes dentales en riesgo de infección}

\section{Antibiotic prophylaxis protocol for dental patients at risk of infection}

\section{Resumen}

La profilaxis antibiótica involucra el uso de fármacos en una etapa preoperatoria de atención odontológica, con la finalidad específica de contribuir a la prevención de infecciones sistémicas como la endocarditis infecciosa (EI) asociada a bacteriemias transitorias por procedimientos odontológicos que implican sangrado en pacientes con alto riesgo de complicación por su condición sistémica cardiaca o no cardiaca. Esta complicación potencial inflama el revestimiento interno de las válvulas cardiacas, por proliferación de microorganismos específicos, y aunque es infrecuente es responsable de una elevada tasa de morbilidad y mortalidad. En el año 2007, la Asociación Estadounidense del Corazón (AHA), publica la última actualización vigente en la literatura sobre las recomendaciones y directrices aplicadas para el uso de profilaxis antibiótica. El objetivo de esta revisión pretende desarrollar una síntesis de evidencia existente seguida de un balance honesto de los riesgos y beneficios de forma individual sobre el uso de este protocolo. Se realizó una búsqueda digital integral en idioma español e inglés sobre los protocolos de profilaxis antibiótica en pacientes con riesgo de infección. Se incluyeron bases de datos como: MEDLINE, PUBMED y SciELO, adicionalmente se tomaron como referentes de partida las recomendaciones de la AHA. Se concluye con gran énfasis y soporte de la literatura actual, que los beneficios del uso de profilaxis antibiótica en un momento preoperatorio superan los posibles riesgos de resistencia bacteriana y anafilaxia, por lo que son totalmente justificables y requeridos para los pacientes adultos y nińos inmunológicamente comprometidos.

Palabras clave: Endocarditis bacteriana; Bacteriemia; Profilaxis antibiótica; Manejo odontológico (fuente: DeCS BIREME).

\begin{abstract}
Antibiotic prophylaxis involves the use of drugs at a preoperative stage of dental care, with a specific aim of contributing to the prevention of systemic infections such as infectious endocarditis (IE) associated with transient bacteremia due to dental procedures that involve bleeding in patients at high risk of complication due to their non-cardiac or cardiac systemic condition. This potential complication inflames the inner lining of the heart valves, due to the proliferation of specific microorganisms, and although it is
\end{abstract}

\section{Artículo de Revisión}

María Isabel Astudillo-Crespo ${ }^{1, a}$, María Cristina Alvear-Córdova ${ }^{1, a, b}$

${ }^{1}$ Universidad Católica de Cuenca, Facultad de Odontología, Cuenca, Ecuador.

a Odontóloga.

${ }^{\mathrm{b}}$ Especialista en Odontopediatría.

\section{Correspondencia:}

María Isabel Astudillo-Crespo: miastudilloc75@est. ucacue.edu.ec

Av. Ordoñez Lasso y Jacarandá 1-27. Sector Oro Verde.

Cuenca. Ecuador

ORCID: 0000-0001-8809-6518

\section{Coautora:}

María Cristina Alvear-Córdova: cristhialvear@hotmail.com ORCID: 0000-0003-4704-4564

\section{Editor:}

Eliberto Ruiz Ramirez

Universidad Nacional Mayor de San Marcos, Perú.

Conflicto de intereses: los autores declaran no tener conflictos de interés.

\section{Fuente de financiamiento: autofinanciado.}

\section{Recibido: 16/08/21}

Aceptado: 28/10/21

Publicado: 21/01/22 
uncommon, it is responsible for a high rate of morbidity and mortality. In 2007, the American Heart Association (AHA) published the last current update in the literature on the recommendations and guidelines applied for the use of antibiotic prophylaxis. The aim of this review is to develop a synthesis of existing evidence followed by an honest assessment of the risks and benefits individually on the use of this protocol. A comprehensive digital search was conducted in both Spanish and English on antibiotic prophylaxis protocols in patients at risk of infection. Databases such as MEDLINE, PUBMED, and SciELO were included, in addition the AHA recommendations were taken as baseline references. It is concluded with great emphasis and support from the current literature, that the benefits of the use of antibiotic prophylaxis in a preoperative moment overcome the possible risks of bacterial resistance and anaphylaxis, so they are fully justifiable and required for immunologically compromised adult and children patients.

Keywords: Bacterial endocarditis; Bacteremia; Antibiotic prophylaxis; Dental management (source: MeSH NLM).

\section{Introducción}

La profilaxis antibiótica (PA) implica el empleo de antibióticos en una etapa denominada preoperatoria, con el objetivo de prevenir infecciones, que surgen de bacteriemias asociadas con procedimientos dentales en pacientes con alto riesgo de complicaciones infecciosas locales (cutáneas, mucosas, óseas y dentales) o sistémicas graves como la endocarditis infecciosa (EI), esta última resulta una condición poco frecuente que afecta aproximadamente alrededor de $5-10$ personas por cada $100000 \mathrm{al}$ año ${ }^{1-3}$; sin embargo, tiene una alta morbilidad y mortalidad no solo hospitalaria sino también en el primer año tras el alta ${ }^{2,3}$. En consecuencia, se vuelve una complicación importante de prevenir con el uso de antibióticos específicos, que ejercen el papel de atacar a los microorganismos durante la fase de contaminación, antes de una posible colonización bacteriana. El fundamento de la profilaxis es reducir o eliminar la bacteriemia transitoria causada tras procedimientos dentales invasivos ${ }^{1}$.

Dentro de este contexto es pertinente destacar que el uso de antibióticos puede resultar en el desarrollo de microorganismos resistentes, por lo que la profilaxis se limita únicamente a aquellos grupos de pacientes en mayor riesgo, evitando un abuso indiscriminado de antimicrobianos. Además, la utilización de PA no proporciona prevención absoluta del desarrollo de una infección. Síntomas posteriores a un procedimiento dental que engloben fiebre, malestar, debilidad y letargo pueden ser el indicio de una insuficiente dosis antibiótica y la necesidad de una evaluación médica adicional ${ }^{4}$.

El origen de la PA se remonta al año de 1923, los autores Lewis y Grant ${ }^{5}$, sugirieron por primera vez que la EI, podría ser causada por bacterias liberadas en la circulación durante un procedimiento dental. Años después en 1941, tuvo lugar el primer uso registrado de profilaxis antimicrobiana, y hasta 1955 la Asociación Americana del Corazón (AHA) emitió las primeras directrices de profilaxis para proteger a los pacientes con cardiopatías reumáticas o congénitas ${ }^{6}$. Desde la emisión de las pautas originales de la AHA, han surgido varias revisiones y desarrollo de diversos lineamientos adaptados a poblaciones locales a nivel del mundo, en el año 2007 se registra la última recomendación publicada por la AHA con sus respectivas recomendaciones para pacientes adultos e infantiles ${ }^{5-6}$. Se han realizado varios tipos de experimentos para intentar determinar la eficacia de la profilaxis antibiótica, entre estos: estudios en animales, estudios de casos y controles, estudios que utilizan bases de datos administrativas antes y después de los cambios a las pautas, así como estudios para determinar el impacto de la profilaxis antes de los procedimientos dentales en personas de riesgo ${ }^{2}$. Sin embargo, no queda claro actualmente si la profilaxis antibiótica es netamente eficaz, continúa siendo un tema dividido, incluso existen escasos ensayos clínicos aleatorizados con el poder estadístico adecuado para ayudar a informar y regular la toma de decisiones en este campo. El objetivo de esta revisión pretende desarrollar una síntesis de la evidencia existente seguida de un balance honesto de los riesgos y beneficios de forma individual. Dado el creciente número de organismos que han desarrollado resistencia a los regímenes antibióticos actuales, así como la posibilidad de una reacción adversa al uso de estos fármacos principalmente anafiláctica, se orienta a la prudencia en el uso de estos en el campo odontológico ${ }^{4-6}$. Este artículo se centra en los criterios de diversas asociaciones: (AHA) Asociación Estadounidense del Corazón, (ADA) Asociación Dental Americana, (AAPD) Academia Estadounidense de Odontología Pediátrica, en relación con el uso de profilaxis antibiótica, con énfasis en la evidencia científica de alto nivel.

\section{Metodología}

La búsqueda bibliográfica integral se basó específicamente en un enfoque cuantitativo de la literatura con diseño de investigación descriptivo. El tipo de búsqueda se realizó mediante tres métodos: (i) Por el ámbito documental, (ii) Con la técnica observacional y (iii) Temporalidad retrospectiva. Se analizó el total de publicaciones relevantes sobre el tema: "Protocolo de profilaxis antibiótica para pacientes dentales en riesgo de infección" detectado entre los últimos 10 años mediante las siguientes bases de datos: MEDLINE, PUBMED, SciELO, SCOPUS, Cochrane library, Google scholar. Se utilizaron palabras clave en inglés y español como: endocarditis bacteriana (IE: Infective Endocarditis), bacteriemia, manejo odontológico, profilaxis antibiótica (AP: 
Antibiotic Prophylaxis), y guías publicadas por asociaciones importantes como la ADA (American Dental Association) y la AHA (American Heart Association). Se recopilaron un total de 74 artículos selectos por criterios de relevancia, título, contenido y fecha sin restricción de idioma. Los artículos excluidos fueron los menos relevantes y desactualizados por lo que se concluyó con un total de 46 publicaciones de importancia para el desarrollo del contenido de la revisión literaria.

\section{Condiciones cardiacas y no cardiacas con alto riesgo de endocarditis infecciosa en las que se recomienda profilaxis antibiótica previa a procedimientos dentales}

La EI, es la inflamación del revestimiento interno de las válvulas y cavidades cardiacas producida por microorganismos que han crecido de una manera significativa, se presenta comúnmente en procedimientos quirúrgicos, independientemente de la afección cardíaca subyacente. Se considera una condición que pone en peligro la vida del paciente ${ }^{7}$. Los avances en la terapia antimicrobiana, la gestión de las complicaciones, junto a la mejora de la tecnología quirúrgica han reducido su morbilidad y mortalidad, sin embargo, numerosos factores de riesgo asociados al paciente como la edad avanzada, la diabetes mellitus, el tratamiento inmunosupresor, y otros, pueden complicar el desarrollo de esta ${ }^{7-8}$. La EI compromete frecuentemente a personas que cursan enfermedades cardiacas o condiciones valvulares específicas, por ello, todos los procedimientos dentales que implique sangrado en este grupo de pacientes se convierten en un potencial riesgo de complicaciones graves ${ }^{8}$. Se ha evidenciado que la presentación de la enfermedad puede ser aguda y progresiva, sin embargo, también puede debutar sin sintomatología específica y la presencia única de febrícula ${ }^{8}$. La mayoría de los pacientes con enfermedades valvulares vigentes, precisan de PA previa a cualquier tipo de procedimiento dental que implica manipulación de los tejidos blandos y subsiguiente sangrado ${ }^{9}$. A lo largo de los años, expertos han analizado las condiciones cardiacas graves con alto riesgo de proliferación bacteriana tras procedimientos clínicos y quirúrgicos, se afirma que los pacientes con prótesis cardiacas valvulares u homoinjertos tienen una prevalencia del $50 \%$ de padecer EI de no ser tratada adecuadamente con una PA. Un estudio realizado en Londres en el año 2017 asegura que de todas las condiciones cardíacas en las que se desarrolla bacteriemia tras procedimientos odontológicos, la cardiopatía congénita y un historial previo de EI, son las de mayor mortalidad ante la colonización de Estreptococos del grupo viridans ${ }^{10}$. Este tipo de microorganismos representa el grupo bacteriano con mayor prevalencia, al formar parte de la flora normal de la piel, cavidad oral y del tracto gastrointestinal, causando al menos $50 \%$ de los casos de EI valvulares ${ }^{9}$. En el año 2007 la Asociación Estadounidense del Corazón (AHA) y el Colegio Americano de Cardiología (ACC) publicaron una actualización enfocada a sus guías anteriores sobre el manejo de las cardiopatías valvulares (Tabla 1), re- forzando sus recomendaciones pasadas, al afirmar que la PA es razonable para el subconjunto de pacientes con condiciones cardiacas asociadas y con alto riesgo de sufrir resultados adversos ${ }^{10}$.

Tabla 1. Condiciones cardiacas asociadas con alto riesgo de endocarditis en las que se recomienda profilaxis antibiótica para procedimientos dentales

\begin{tabular}{|c|}
\hline $\begin{array}{c}\text { I. Prótesis valvulares, incluidas las prótesis transcatéteres y homoin- } \\
\text { jertos. Material protésico utilizado para la reparación de válvulas } \\
\text { cardíacas, como anillos y cuerdas de anuloplastia. }\end{array}$ \\
\hline ll. Historial de endocarditis infecciosa previa. \\
III. Enfermedad cardiaca congénita. \\
\hline a. Cardiopatía congénita cianótica o isquémica no reparada. \\
\hline $\begin{array}{c}\text { b. Cardiopatía congénita cianótica completamente reparada con } \\
\text { material protésico o dispositivos, durante los primeros seis meses } \\
\text { tras el procedimiento. }\end{array}$ \\
\hline $\begin{array}{c}\text { c. Cardiopatía congénita reparada con defectos residuales adyacen- } \\
\text { tes en el lugar del dispositivo protésico. }\end{array}$ \\
\hline IV. Trasplante cardiaco con desarrollo de valvulopatía. \\
\hline
\end{tabular}

Adaptación de la fuente: Asociación Estadounidense del Corazón, Inc. ${ }^{9}$

Las series publicadas sobre EI en pacientes con cardiopatía isquémica no tienen la potencia suficiente para determinar hasta qué punto una forma específica de cardiopatía isquémica es un factor de riesgo independiente de morbilidad y mortalidad ${ }^{9-10}$. No obstante, la mayoría de las series retrospectivas sugieren que los pacientes con cardiopatías cianóticas complejas y los que tienen derivaciones paliativas postoperatorias, conductos u otras prótesis tienen un alto riesgo de contraer EI a lo largo de su vida ${ }^{10}$. Otras revisiones informaron que la presencia de material protésico y la cardiopatía cianótica compleja en pacientes de muy corta edad (hasta 2 años), son dos factores que se asocian a peores pronósticos de la EI, sin embargo, algunos tipos de cardiopatías pueden repararse completamente sin defectos cardíacos residuales ${ }^{11}$. Cuando se trata de procedimientos dentales en pacientes pediátricos, la PA es recomendada solo al existir un riesgo muy alto de presentar consecuencias desfavorables debido a una EI ${ }^{12}$.

La Academia Estadounidense de Odontología Pediátrica (AAPD), deduce que no es posible pronosticar cuándo un paciente pediátrico es susceptible a desencadenar una infección, sin embargo, la inmunosupresión, las enfermedades cardiacas y las inmunodeficiencias del paciente, pueden impedir su capacidad de batallar una simple infección ${ }^{12}$. En niños de riesgo, el uso de PA no brinda una prevención absoluta de la infección, incluso se puede desencadenar una insuficiencia antibiótica debido a los síntomas posteriores del procedimiento de infección aguda, por lo que siempre se va a requerir de una revisión médica adicional ${ }^{12}$. Para aminorar el riesgo de desarrollar resistencia antibiótica o reacción anafiláctica en niños, el uso conservador de fármacos es el más indicado para la prevención de EI ${ }^{12}$. En los pacientes con afecciones cardiacas la EI constituye una condición mortal y poco común con una incidencia de entre 0,05 
y 0,12 casos por cada 1000 niños aproximadamente ${ }^{12}$ 13. Los antibióticos son recomendados en todos los procedimientos dentales que involucren manipulación del tejido gingival, región periapical y perforación de mucosa para los nińos que padecen enfermedades cardiacas ${ }^{12-13}$. Es de suma importancia la motivación de los padres hacia sus hijos para mantener una higiene oral óptima en pacientes comprometidos que tienen un alto riesgo de desarrollar EI.

Se han definido también las condiciones no cardiacas con alto riesgo de padecer EI, para las cuales se recomienda una PA ante procedimientos dentales, estas abarcan a los pacientes adultos con enfermedades graves que requieren un cuidado especial previo a procedimientos quirúrgicos dentales que impliquen altas posibilidades de sangrado ${ }^{13}$ (Tabla 2). Actualmente, las guías publicadas por la AHA y ADA aseguran que se debe dar la misma importancia a las condiciones tanto cardiacas y no cardiacas, ya que estas últimas abarcan enfermedades graves que requieren de un régimen profiláctico estricto ${ }^{13}$.

Tabla 2. Condiciones no cardiacas asociadas con alto riesgo de endocarditis en las que se recomienda profilaxis antibiótica para procedimientos dentales

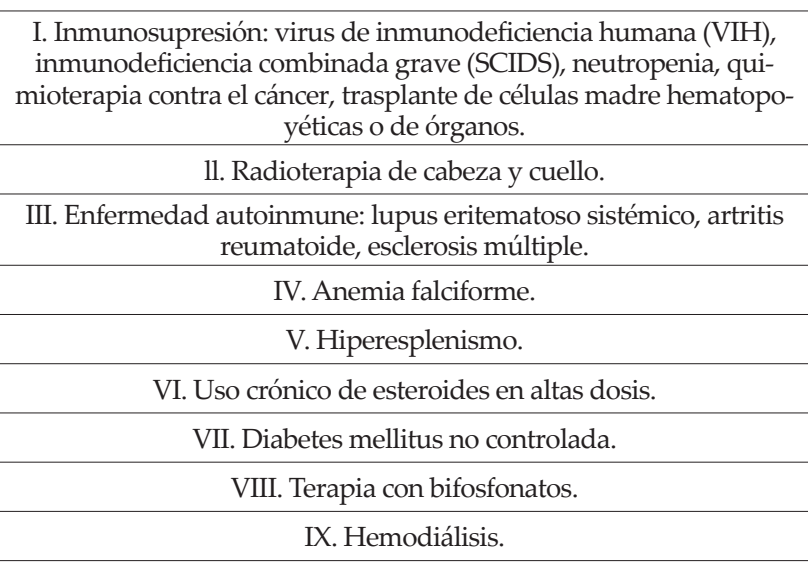

Adaptación de la fuente: Asociación Estadounidense del Corazón, Inc. ${ }^{13}$

\section{Especies bacterianas implicadas en el desarrollo de infecciones posoperatorias}

La capacidad de las diversas especies bacterianas para adherirse a sitios específicos determina la localización anatómica de la infección causada por esos microorganismos ${ }^{14}$. Los mediadores de la adhesión bacteriana sirven como factores de virulencia en la patogénesis de la endocarditis infecciosa $13^{13-14}$. Se ha demostrado en modelos animales de endocarditis experimental que los componentes de la superficie de las bacterias presentes en los estreptococos, estafilococos y enterococos funcionan como adherencia crítica ${ }^{14}$. Algunos estreptococos del grupo de los viridans contienen una proteína que es antígeno receptor de lipoproteínas que sirve como una importante adherencia a la matriz plaquetaria ${ }^{13}$. Las adherencias de Estafilococos funcionan al menos de dos maneras, en una los componentes microbianos de la superficie reconocen las moléculas de la matriz, facilitando la adhesión de los estafilococos a los dispositivos médicos que se recubren con las proteínas de la matriz después de su implantación ${ }^{15}$. En la otra, las estructuras extracelulares bacterianas contribuyen a la formación de una biopelícula que se forma en la superficie de los dispositivos médicos implantados. En ambos casos las adherencias de estafilococos son importantes factores de virulencia ${ }^{14-16}$.

Son varias las bacterias que pueden llegar a ser mortales al momento de proliferar y crear infecciones posoperatorias, dentro de los principales microorganismos comúnmente encontrados, se han evidenciado los estreptococos del grupo viridans, estos microorganismos son habitantes frecuentes de la cavidad oral y forman parte del $30 \%$ de la flora del surco gingival, causando el $50 \%$ total de los casos de EI. Solo el 1\% de los casos presentados de EI son originados tras procedimientos dentales ${ }^{17}$. Otra especie implicada en el desencadenamiento de EI es el estafilococos aureus, los individuos que han consumido drogas y los que padecen cierta resistencia ante fármacos, son más susceptibles a una proliferación bacteriana de este tipo ${ }^{18}$. En pacientes pediátricos no es muy común el desencadenamiento de EI, sin embargo, los microorganismos que se han repetido en varias investigaciones de prevención de EI en niños con afecciones cardiacas son enterococo y estafilococos aureus, definiéndolos como predictores independientes de mortalidad ${ }^{19-20}$. En la EI por estreptococos del grupo viridans o enterococos de la prótesis cardiaca valvular, el espectro de la enfermedad puede ir desde una infección relativamente benigna hasta la disfunción valvular grave, insuficiencia cardíaca, y múltiples eventos embólicos hasta la muerte $21-23$.

\section{Régimen profiláctico antimicrobiano para procedimientos odontológicos}

El régimen antibiótico de las distintas guías existentes maneja modificaciones similares, sin embargo, las consideraciones profilácticas que se recomiendan por la AHA son las más utilizadas ${ }^{24}$. Esta guía da prioridad a la vía de administración oral, lo cual favorece su cumplimiento. En la Tabla 3 se presentan los regímenes profilácticos según su vía de administración, una hora antes del proceso odontológico que pueda implicar riesgo ${ }^{25-26}$. El fármaco de primera elección es la Amoxicilina por vía oral, se administra de 30 a 60 minutos antes del procedimiento con una dosis de $2 \mathrm{~g}$ en adultos y $50 \mathrm{mg} / \mathrm{kg}$ en niños ${ }^{27}$. Le sigue la clindamicina como fármaco de segunda elección en el caso de padecer alguna reacción alérgica ante la Amoxicilina, con su respectiva dosis de $600 \mathrm{mg}$ por vía oral en adultos y $20 \mathrm{mg} / \mathrm{kg}$ en niños. Si existe alguna incapacidad de administrar el fármaco por la vía oral, también se dan opciones por la vía intramuscular o intravenosa, recetando ampicilina de $2 \mathrm{~g}$ en adultos y $50 \mathrm{mg} / \mathrm{kg}$ en niños ${ }^{27-28}$. Se ha definido que cuando se trata a pacientes con tratamiento inmunosupresor, la clindamicina puede ser la primera opción ya que este antimicrobiano posee un espectro extenso ante bacterias anaerobias grampositivas, reforzando acciones 
de fagocitosis, haciéndolo ideal para pacientes con este tipo de condiciones ${ }^{29-30}$.

\section{Procedimientos dentales que requieren profilaxis antibiótica}

Son varios los procesos odontológicos que implican extrema manipulación del tejido gingival o región periapical del diente, en los que la profilaxis antibiótica es necesaria para el desarrollo correcto del proceso preoperatorio, operatorio e incluso el postoperatorio, la precaución por parte del profesional y conocimiento previo de la historia clínica del paciente comprometido llevará al éxito del tratamiento, evitando desencadenar afecciones que pueden aparecer por una proliferación bacteriana $^{31-32}$.

En los últimos años, se ha señalado que una fuente potencial de ciertas infecciones probablemente deriva de procedimientos bucales que, al generar sangrado, permiten la introducción de los microorganismos al torrente sanguíneo, provocando efectos sistémicos y contribuyendo al desarrollo de enfermedades como la endocarditis infecciosa, de modo que, el estado físico desfavorable de la persona beneficia el desarrollo de infecciones producidas por este tipo de bacterias ${ }^{33-34}$. Se afirma que la bacteriemia transitoria es común con la manipulación de los dientes y tejidos periodontales, sin embargo, existe una amplia variación en las frecuencias reportadas de bacteriemia en los pacientes como resultado de procedimientos dentales ${ }^{35-38}$. En la Tabla 4 se presentan los procedimientos odontológicos que constituyen factores de riesgo de infección focal y sistémica en pacientes con condiciones sistémicas asociadas. Se define que aquellos procedimientos que requieren de extrema manipulación y sangrado representan significativamente un mayor riesgo ante la proliferación bacteriana ${ }^{39-40}$. Las extracciones dentales y cirugías maxilofaciales cuentan con una alta incidencia de contraer una posible infección ante bacteremia, mientras que otros procedimientos como la colocación de grapas e impresiones dentales tienen un menor porcentaje de riesgo de bacteremia en pacientes con EI ${ }^{41-44}$.

Cabe recalcar, que el profesional odontológico, según su criterio basado en la historia clínica y antecedentes médicos del paciente, definirá si es necesaria una Profilaxis Antibiótica, sin embargo, es de suma importancia tomar en cuenta los lineamientos decretados y actualizados por organizaciones mundiales como son la AHA y $\mathrm{ADA}^{45-46}$.

Tabla 3. Regímenes de profilaxis antibiótica a emplear en procedimientos dentales según la AHA

\begin{tabular}{cccc}
\hline & & Régimen: Una sola dosis de $\mathbf{3 0}$ a $\mathbf{6 0}$ minutos antes del procedimiento dental \\
\hline Situación & Agente & Niños & Adultos \\
\hline Oral & Amoxicilina & $50 \mathrm{mg} / \mathrm{kg}$ & $2 \mathrm{~g}$ \\
\hline No se puede tomar el medicamentooral & Ampicilina & $50 \mathrm{mg} / \mathrm{kg} \mathrm{IM} \mathrm{o} \mathrm{IV}$ & $2 \mathrm{~g} \mathrm{IM} \mathrm{o} \mathrm{IV}$ \\
& Cefazolina & $50 \mathrm{mg} / \mathrm{kg} \mathrm{IM} \mathrm{o} \mathrm{IV}$ & $1 \mathrm{~g} \mathrm{IM}$ 0 IV \\
\hline Alérgicos a penicilinas o ampicilina oral & Cefalexina & $50 \mathrm{mg} / \mathrm{kg}$ & $2 \mathrm{~g}$ \\
& Clindamicina & $20 \mathrm{mg} / \mathrm{kg}$ & $600 \mathrm{mg}$ \\
& Azitromicina & $15 \mathrm{mg} / \mathrm{kg}$ & $500 \mathrm{mg}$ \\
\hline Alérgicos a penicilinas o ampicilina e & Cefazolina & $50 \mathrm{mg} / \mathrm{kg} \mathrm{IM} \mathrm{o} \mathrm{IV}$ & $1 \mathrm{~g} \mathrm{IM} \mathrm{o} \mathrm{IV}$ \\
\hline incapaz de tomar el medicamento oral & Clindamicina & $20 \mathrm{mg} / \mathrm{kg} \mathrm{IM} \mathrm{o} \mathrm{IV}$ & $600 \mathrm{mg} \mathrm{IM} \mathrm{o} \mathrm{IV}$ \\
\hline
\end{tabular}

Adaptación de la fuente. Asociación Dental Americana ${ }^{30}$

Tabla 4. Factores de riesgo de infección focal y sistémica en pacientes con condiciones sistémicas asociadas

\begin{tabular}{cccc}
\hline Procedimientosodontológicos de alto riesgo & \multicolumn{2}{c}{ Incidencia } & Procedimientosodontológicos de bajo riesgo \\
\hline Extracciones & $80 \%$ & $10 \%$ & Manipulación de grapas para aislamiento absoluto \\
Implantes & $70 \%$ & $20 \%$ & Profilaxis Dental \\
Drenajes & $60 \%$ & $40 \%$ & Sondaje Periodontal \\
Biopsias & $75 \%$ & $30 \%$ & Retiro de puntos o suturas \\
Injertos óseos & $80 \%$ & $35 \%$ & Remoción de implantes \\
Cirugía periodontal & $70 \%$ & $20 \%$ & Endodoncia \\
Raspado y alisado radicular & $50 \%$ & $25 \%$ & Colocación de aparatos ortodónticos \\
Gingivectomía & $60 \%$ & $5 \%$ & Colocación de matriz e hilo retractor \\
Tallado que incluyasangre & $40 \%$ & $20 \%$ & Anestesia troncular \\
Cirugía maxilofacial & $80 \%$ & $5 \%$ & Impresiones dentales \\
\hline
\end{tabular}

Adaptación de la fuente: Asociación Estadounidense del Corazón, Inc. ${ }^{36}$ 


\section{Conclusiones}

Según la revisión exhaustiva de literatura actualizada, se concluye que la profilaxis antibiótica tiene como finalidad prevenir el desencadenamiento de una endocarditis infecciosa, de tal manera que no exista proliferación bacteriana. Para indicar PA, debe existir un riesgo considerable proporcionado por el estado sistémico del paciente (tratamiento inmunosupresor o afecciones cardíacas subyacentes). El fármaco de elección debe poseer un espectro extenso ante las bacterias implicadas, según varios estudios se deduce que la amoxicilina vía oral de $2 \mathrm{~g}$ en adultos y $50 \mathrm{mg} / \mathrm{kg}$ en niños es la primera opción para pacientes con cardiopatías valvulares, seguido por la clindamicina con su respectiva dosis de $600 \mathrm{mg}$ por vía oral en adultos y $20 \mathrm{mg} / \mathrm{kg}$ en niños. Para administrar profilaxis antibiótica se requieren razones científicas afianzadas y con un protocolo médico legal. Por lo tanto, es de suma importancia tener en cuenta que, al tratarse de procedimientos dentales con riesgo de infección, el odontólogo será responsable del tratamiento dental del paciente y según su historia médica, tomar la mejor decisión, basándose en los beneficios del paciente y evitando cualquier tipo de contraindicación o reacción que sea perjudicial en el futuro. Este artículo de revisión se apoya específicamente en las recomendaciones guiadas por la AHA y ADA, las cuales fueron diagramadas y citadas respectivamente debido a su alto éxito ante la prevención de EI.

\section{Referencias bibliográficas}

1. Mougeot FK, Saunders SE, Brennan MT, Lockhart PB. Associations between bacteremia from oral sources and distant-site infections: tooth brushing versus single tooth extraction. Oral Surg Oral Med Oral Pathol Oral Radiol. 2015;119(4):430-5.

2. Dayer M, Thornhill M. Is antibiotic prophylaxis to prevent infective endocarditis worthwhile?. J Infect Chemother. 2018;24(1):18-24.

3. Singh Gill A, Morrissey H, Rahman A. A Systematic Review and Meta-Analysis Evaluating Antibiotic Prophylaxis in Dental Implants and Extraction Procedures. Medicina. 2018;54(6):95. DOI: 10.3390/medicina54060095.

4. American Academy of Pediatric Dentistry. Antibiotic prophylaxis for dental patients at risk for infection. The Reference Manual of Pediatric Dentistry. Chicago, Ill.: American Academy of Pediatric Dentistry; 2021:465-70.

5. Lewis, Grant, Isola G, Alibrandi A, Pedulla E, Grassia V. Analysis of the Effectiveness of Lornoxicam and Flurbiprofen on Management of Pain and Sequelae Following Third Molar Surgery: A Randomized, Controlled, Clinical Trial. J. Clin. Med. 2019;8(3):325. DOI: 10.3390/ jcm8030325.

6. Milani B, Bauer H, Sampaio F, Horliana A, Perez F, Tortamano I, Jorge W. Antibiotic therapy in fully impacted lower third molar surgery: randomized three arm, double-blind, controlled trial. Oral Maxillofac. Surg. 2015;19:341-346.
7. Nishimura RA, Otto CM, Bonow RO, Carabello BA, Erwin JP, et al. 2017 AHA/ACC Focused Update of the 2014 AHA/ACC Guideline for the Management of Patients With Valvular Heart Disease: A Report of the American College of Cardiology/American Heart Association Task Force on Clinical Practice Guidelines. Circulation. 2017;135(25):22-34.

8. ADA. Antibiotic prophylaxis prior to dental procedures. Oral Health Topics. 2017;1(2):5-10.

9. Asociación Estadounidense del Corazón. Inc. Circulación. 2017;116:1736-1754.

10. Habib G, Lancellotti P, Antunes MJ, Bongiorni MG, Casalta JP, Del Zotti F, et al. 2015 ESC Guidelines for the management of infective endocarditis: The Task Force for the Management of Infective Endocarditis of the European Society of Cardiology (ESC). EurHeart J. 2015;36:3075-123.

11. Roberts GJ, Jaffrey EC, Spract DA, Petrie A, Greville C, Wilson M. Duration, prevalence and intensity of bacteremia after dental extractions in children. Heart. 2016;92(9):12-7.

12. Academia Estadounidense de Odontología Pediátrica. Profilaxis antibiótica para pacientes dentales con riesgo de infección. El Manual de referencia de odontología pediátrica. Chicago: Academia Estadounidense de Pediatría Odontología; 2020: 447-52.

13. Asociación Estadounidense del Corazón, Inc. Circulación. 2017;116:1736-1754.

14. American Academy of Pediatric Dentistry. Antibiotic prophylaxis for dental patients at risk for infection. Pediatr Dent. 2014. (2016 Update); 36:287-92.

15. Cahill TJ, Dayer M, Prendergast B, Thornhill M. Do patients at risk of infective endocarditis need antibiotics before dental procedures? BMJ. 2017;358:j3942. DOI: 10.1136/bmj.j3942.

16. Bläckberg A, Nilson B, Özenci V, et al. Infective endocarditis due to Streptococcus dysgalactiae: clinical presentation and microbiological features. Eur J Clin Microbiol Infect Dis. 2018;37(12):2261-2272.

17. Lindell F, Söderquist B, Sundman K, et al. Prosthetic valve endocarditis caused by Propionibacterium species: A national registry-based study of 51 Swedish cases. Eur J Clin Microbiol Infect Dis. 2018;37(4):765-771.

18. Mackie AS, Liu W, Savu A, et al. Infective endocarditis hospitalizations before and after the 2007 American heart association prophylaxis guidelines. Can J Cardiol. 2016;32(8):942-948.

19. Tate AR, Norris CK, Minniti CP. Antibiotic prophylaxis for children with sickle cell disease: A survey of pediatric dentistry residency program directors and pediatric hematologists. Pediatr Dent. 2016;28(3):2-5.

20. Arduino PG, Tirone F, Schiorlin E, Esposito M. Single preoperative dose of prophylactic amoxicillin versus a 2-day postoperative course in dental implant surgery. A two-Centre randomised controlled trial. Eur J Oral Implantol. 2015;8:143-9.

21. General Assembly of the World Medical Association. World Medical Association Declaration of Helsinki: 
ethical principles for medical research involving human subjects. J Am Coll Dent. 2018;81(3):13-80.

22. National Institute for Health and Care Excellence (NICE). Prophylaxis against infective endocarditis: antimicrobial prophylaxis against infective endocarditis in adults and children undergoing interventional procedures [Internet]. National Institute for Health and Clinical Excellence; 2008 [citado el 08 de julio 2016]. Disponible en: http://www.nice.org.uk/CG64.

23. Conly J. Antimicrobial resistance: revisiting the "tragedy of the commons". Bull World Health Organ. 2010;88(11):805-6.

24. Wilson WR, Jaumin PM, Danielson GK, Giuliani ER, Washington JA II, Geraci JE. Prosthetic valve endocarditis. Ann Intern Med. 2017;82:751-756.

25. Prendergast BD. Infective endocarditis. Lancet. 2016;387:882-93.

26. Ashrafian H, Bogle RG. Antimicrobial prophylaxis for endocarditis: emotion or science?. Heart. 2017;93:5- 6.

27. Taubert K, Wilson W. Interactive clinical case: To give, or not to give, infective endocarditis prophylaxis. Approach 1: Prophylaxis not recommended. BMJ Blogs. Heart Asia. 2017;9(1):27-29.

28. Lockhart PB, Durack DT. Oral microflora as a cause of endocarditis and other distant site infections. Infect Dis Clin North Am. 2016;13:833-850.

29. Thornhill MH, Dayer $H$, et al. Quantifying infective endocarditis risk in patients with predisposing cardiac conditions. Eur Heart J. 2018;39(7):586-95.

30. Asociación Dental Americana. J Am Dent. Elseiver. 2015;146(1):11-16

31. Gould FK, Elliott TS, Foweraker J, Fulford M, Perry JD, Roberts GJ, et al. Working Party of the British Society for Antimicrobial Chemotherapy. Guidelines for the prevention of endocarditis: Report of the Working Party of the British Society for Antimicrobial Chemotherapy. J Antimicrob Chemother. 2006 (2020 Update); 57:1035-42.

32. NICE. Prophylaxis Against Infective Endocarditis: Antimicrobial Prophylaxis Against Infective Endocarditis in Adults and Children Undergoing Interventional Procedures. Nice Clinical Guidelines No. 64. London: National Institute for Health and Clinical Excellence; 2008 (2019 Update).

33. National Institute for Health and Care Excellence London. Antimicrobial prophylaxis against infective endocarditis in adults and children undergoing interventional procedures. NICE Clinical Guidelines, No. 64. 2018;20(1):2-6.
34. Sollecito TP, Abt E, Lockhart PB, et al. The use of prophylactic antibiotics prior to dental procedures in $\mathrm{pa}$ tients with prosthetic joints: evidence-based clinical practice guideline for dental practitioners - a report of the American Dental Association Council on Scientific Affairs. J Am Dent Assoc. 2015;146(1):11-16.

35. Jones S, Prendergast B, Baddour LM, Lockhart PB, Thornhill $\mathrm{MH}$. Incidence of infective endocarditis in England, 2000-13: a secular trend, interrupted time- series analysis. Lancet. 2015;385(9974):1219-28.

36. Díaz L, Castellanos J. Prevención de Endocarditis Infecciosa en odontología. Adaptado de: Asociación Estadounidense del Corazón. 2017;117(24):3118-25.

37. Dayer $\mathrm{H}$, Thornhill $\mathrm{MH}$, et al. Is antibiotic prophylaxis to prevent infective endocarditis worthwhile? J Infect Chemother. 2018;24(1):18-24.

38. Crasta K, Daly CG, Mitchell D, Curtis B, Stewart D, Heitz-Mayfield LJ. Bacteraemia due to dental flossing. J. Clin Periodonto. 2016;36:323-32.

39. Barbosa M, Prada-López I, Álvarez M, Amaral B, de los Angeles Casares-De-Cal M, Tomás I. Post-Tooth Extraction Bacteraemia: A Randomized Clinical Trial on the Efficacy of Chlorhexidine Prophylaxis. PLoS ONE. 2015;10(5):1-15.

40. Daly CG. Antibiotic prophylaxis for dental procedures. Aust Prescr. 2017;40(5):184-8.

41. Guideline on antibiotic prophylaxis for dental patients at risk for infection. American Academy of Pediatric Dentistry. Pediatr Dent. 2016;38(6):328-33.

42. Guidelines for the management of Infective endocarditis. European Society of Cardiology. Eur Heart J. 2015;36:75-123.

43. Maestre Vera JR, Mateo Maestre M, Sánchez Santana P. Bacteriemia secundaria a procedimientos odontológicos periodontales. Rev Esp Quimioter. 2017;21(3):15-19.

44. Beacher N, Sweeney MP, Bagg J. Dentists, antibiotics and Clostridium difficile-associated disease. Br Dent J. 2015;219(6):275-9.

45. Hunter W. Oral sepsis as a cause of disease. Br Med J. 2019;2065(2):215-216.

46. Koulocheris P, Bergin S, Cryan B, Flanagan O. Intracranial abscess secondary to dental infection. J Ir Dent Assoc. 2018;60:32-34. 